Éléments de calcul vectoriel avec de nombreuses applications à la géométrie, à la mécanique et à la physique mathématique par C. Burali-Forti et R. Marcolongo, traduit de l'italien par S. Lattès. Paris, A. Hermann et fils, 1910. VII + 229 pages. Prix 8 francs.

Diese französische Übersetzung des oben besprochenen Werkes unterscheidet sich von dem italienischen Original im wesentlichen durch einen längeren Zusatz, der die Größen höherer Stufe Graßmanns und die Quaternionen Hamiltons in das System der Vektoranalysis einordnen soll. Ein solcher Versuch ist gewiß interessant; natürlicher und logischer dürfte aber doch der umgekehrte Weg sein.

Schließlich sei noch ein störendes Versehen angeführt: auf pag. 204 fehlt auf der rechten Seite der ersten Gleichung (Zeile 2 v. v.) das Glied - $(V \beta \times V a) x$ und die zum Verständnis dieser Stelle notwendige Bemerkang. ,$(x \times V \beta) V \alpha=(V \beta \wedge V \alpha) \wedge x$, weil $x \perp V \alpha$ ist" ; in der zweiten Gleichung. fehlt in Zeile 5 v. o. innerhalb der zweiten Klammer $+V \beta \wedge V \alpha$.

\title{
H. Rothe.
}

Die Resonanztheorie des Hörens. Als Beitrag zur Lehre von den Tonempfindungen von Dr. Erich $W$ a etzman $\ddot{n}$, Privatdozent für Physik an der Universität Breslau; mit 33 eingedruckten Abbildungen. Braunschweig, Friedrich Vieweg u. Sohn. Geheftet Mk. 5; - 162 Seiten.

In.letzter Zeit erscheint erfreulicherweise das Interesse an akustischen Problemen wieder zugenommen zu haben, denn in kurzer Aufeinanderfolge sind eine Anzahl von guten Büchern über diese gegenüber den neuesten Gebieten arg vernachlässigte Disziplin der Physik herausgekommen. Dazu gehört das Waetzmannsche vor kurzem erschienene Buch. Der Verfasser, der selbst: viel auf diesem Gebiet gearbeitet hat, kann des Dankes vielex Fachkollegen and wohl auch Laien gewiß sein, denn eine zusammenfassende Darstellung der dominierenden Helmholtszchen Hörtheorie war dringendes Bedürfnis geworden. Nach einer den ersten Abschnitt umfassenden und mehr für den Nichtphysiker bestimmten Einleitung, die die physikalischen Grundtatsachen, also Wellen und Schwingangen and ihre Verwertung in der Lehre vom Schall leicht verständlich darstellt, gibt der Verfasser im II. Abschnitt die auf dem anatomischen Bau des Ohres fufende Resonanztheorie von Helmholtz; die Einwände gegen sie, sowie deren Widerlegungen und die positiven Beweise werden im 3. und 4. Kapitel dieses Abschnittes, betrefiend „die physikalischen Eigenschaften der Resonatoren im Ohr" und die "physiologischen Untersuchungen zur Resonanztheorie", eingehend besprochen. Im 3. und 4. Abschnitt wird gezeigt, wie diese Theorie für die Erklärung der akustischen Erscheinungen, für die Klangfarbe, Schwebungen, Konsonanz und Dissonanz, sowie alle Arten der Kombinations- und Unterbrechungstöne ausreicht. Im 5. Abschnitt endlich sind eine Anzahl von hiehergehörigen mathematischen Ergänzungen angeführt. Das Buch ist klar und leicht verständlich geschrieben und vor allem sind viele der neuesten and in oft schwer zugänglichen Zeitschriften niedergelegten Untersuchungen mitberücksichtigt und besprochen. Die unverdientermaßen insbesondere in weiteren Kreisen so wenig bekannte Helmholtz- 
sche Resonanztheorie, die doch eine der schönsten Arbeitshypothesen der Physik überhaupt sein dürfte, wird vielleicht durch dieses Buch, das allen Fachleuten und gebildeten Laien wärmstens empfohlen sei, weitere Verbreitung finden.

K. W. F. Kohlrausch.

Aus Natur und Geisteswelt. Band 304. Die Mechanik der flüssigen Körper von A. v. Thering. 34 Figuren, 112 Seiten, B. G. Teubner, Leipzig 1912.

Das Bändchen schlieBt sich in der Behandlungsweise ganz der „Mechanik fester Körper" desselben Autors an. Die klar und gemeinverständlich mit einem Minimum mathematischen Aufwandes behandelten Sätze werden stets durch numerische Beispiele erläutert und es werden überall die Anwendungen in der Technik und praktische Verhältnisse berücksichtigt.

K. Priz.

The Dynamics of particles and of rigid elastic and fluid bodies, being lectnres on mathematical physies by Arthur, Gordon Webster. 2. Auflage. XII +588 Seiten. Aus B. G. Teubners Sammluug von Lehrbüchern auf dem Gebiete der mathematischen Wissenschaften mit Einschluf ihrer Anwendungen. Band XI. B. G. Teubner, Leipzig 1912. M. 14.

Die zweite Auflage ist ein fast unveränderter Abdruck der ersten. Das innerhalb kurzer Zeit bei einem ein so spezielles Gebiet behandelnden Werke eine neue Auflage nötig wurde, spricht besser als jedes W'ort für die besondere Gediegenheit des Buches.

St, $M$.

Grandzüge der Experimentalphysik, von Prof. Hermann Roth; F. Grub, Stuttgart 1912. Mark 1,60.

Das Büchlein bringt auf dem engen Raum von 111 Druckseiten in gedrängtester Form eine monumentale Fülle von Unrichtigkeiten. Dies ist um so bedenklicher, als der Autor, Lehrer an einer höheren Mädchenschule in Stuttgart, das Buch als erste Einführung in das Physikstudium für Schüler und Schülerinnen von 14 bis 16 Jahren gedacht hat, die dem Unsinn völlig machtlos gegenüberstehen. Was soll sich so ein armer Junge denken, wenn er hört, das die Schwere „ihren Sitz im Erdmittelpunkt hat"? Oder wenn er die kurze, aber unverständliche Definition liest: „Im Schiffsbau bezeichnet man den Schnittpunkt der Wirkungslinie des Auftriebes mit der Symmetriebene des Schiffes als Metazentrum" (pag. 20). „Durchscheinend ist ein Stoff, wenn er nur einen Teil des Lichtes hindurchläßt" (pag. 45).

Man könnte vielleicht geneigt sein, solche Dinge als lediglich unpräzise Ausdrucksweise zu entschuldigen. Aber man bekommt noch ganz andere Dinge zu hören, z. B. daß Olaf Römer die Lichtgeschwindigkeit aus der Wahrnehmung bestimmte, "daß die Verfinsterung" ("welche ein Jupitertrabant erfährt, wenn er darch den Kernschatten des Planeten hindurchgehen muB") "ungefähr 1000 Sekunden länger dauerte, wenn die Erde in Jupiterferne $E_{3}$, als wenn sie sich inihrer Bahn in Jupiternähe $E_{1}$ befand" (pag. 47, mit Figur!).

$E_{s}$ ist eine Kinderstubenvorstellung, daß ein Magnet gesättigt ist, wenn er sein eingenes Gewicht zu tragen vermag. (pag. 82.) 